Negative Plasma Densities Raise Questions

A. Hazi

February 2, 2006 
This document was prepared as an account of work sponsored by an agency of the United States Government. Neither the United States Government nor the University of California nor any of their employees, makes any warranty, express or implied, or assumes any legal liability or responsibility for the accuracy, completeness, or usefulness of any information, apparatus, product, or process disclosed, or represents that its use would not infringe privately owned rights. Reference herein to any specific commercial product, process, or service by trade name, trademark, manufacturer, or otherwise, does not necessarily constitute or imply its endorsement, recommendation, or favoring by the United States Government or the University of California. The views and opinions of authors expressed herein do not necessarily state or reflect those of the United States Government or the University of California, and shall not be used for advertising or product endorsement purposes.

This work was performed under the auspices of the U.S. Department of Energy by University of California, Lawrence Livermore National Laboratory under Contract W-7405-Eng-48. 


\section{Negative Plasma Densities Raise Questions}

N EARLY all the matter encountered on Earth is either a solid, liquid, or gas. Yet plasma - the fourth state of matter-comprises more than 99 percent of the visible universe. Understanding the physical characteristics of plasmas is important to many areas of scientific research, such as the development of fusion as a clean, renewable energy source.

Lawrence Livermore scientists study the physics of plasmas in their pursuit to create fusion energy, because plasmas are an integral part of that process. When deuterium and tritium are heated to the extreme temperatures needed to achieve and sustain a fusion reaction (about 100 million degrees), the electrons in these light atoms become separated from the nuclei. This process of separation is called ionization, and the resulting collection of negatively charged free electrons and positively charged nuclei is known as a plasma. Although plasmas and gases have many similar properties, plasmas differ from gases in that they are good conductors of electricity and can generate magnetic fields.

For the past decade, $x$-ray laser interferometry has been used in the laboratory for measuring a plasma's index of refraction to determine plasma density. (The index of refraction for a given material is defined as the wavelength of light in a vacuum divided by the wavelength of light traveling through the material.) Until now, plasma physicists expected to find an index of refraction less than one. Researchers from Livermore and Colorado State University recently conducted experiments on aluminum plasmas at the Laboratory's COMET laser facility and observed results in which the index of refraction was greater than one.

This surprising result implied a negative electron density. Livermore physicist Joseph Nilsen and his colleagues from Livermore and the University of Notre Dame have performed sophisticated calculations to explain this phenomenon. Previously, researchers believed that only free electrons contributed to the index of refraction. Nilsen and his colleagues posit that bound electrons attached to the ions in plasmas can greatly affect the index of refraction and make it greater than one. Furthermore, if the effect of bound electrons is ignored when analyzing experimental results from $\mathrm{x}$-ray interferometry, the electron density of plasmas may be indeterminate or significantly under- or overestimated.
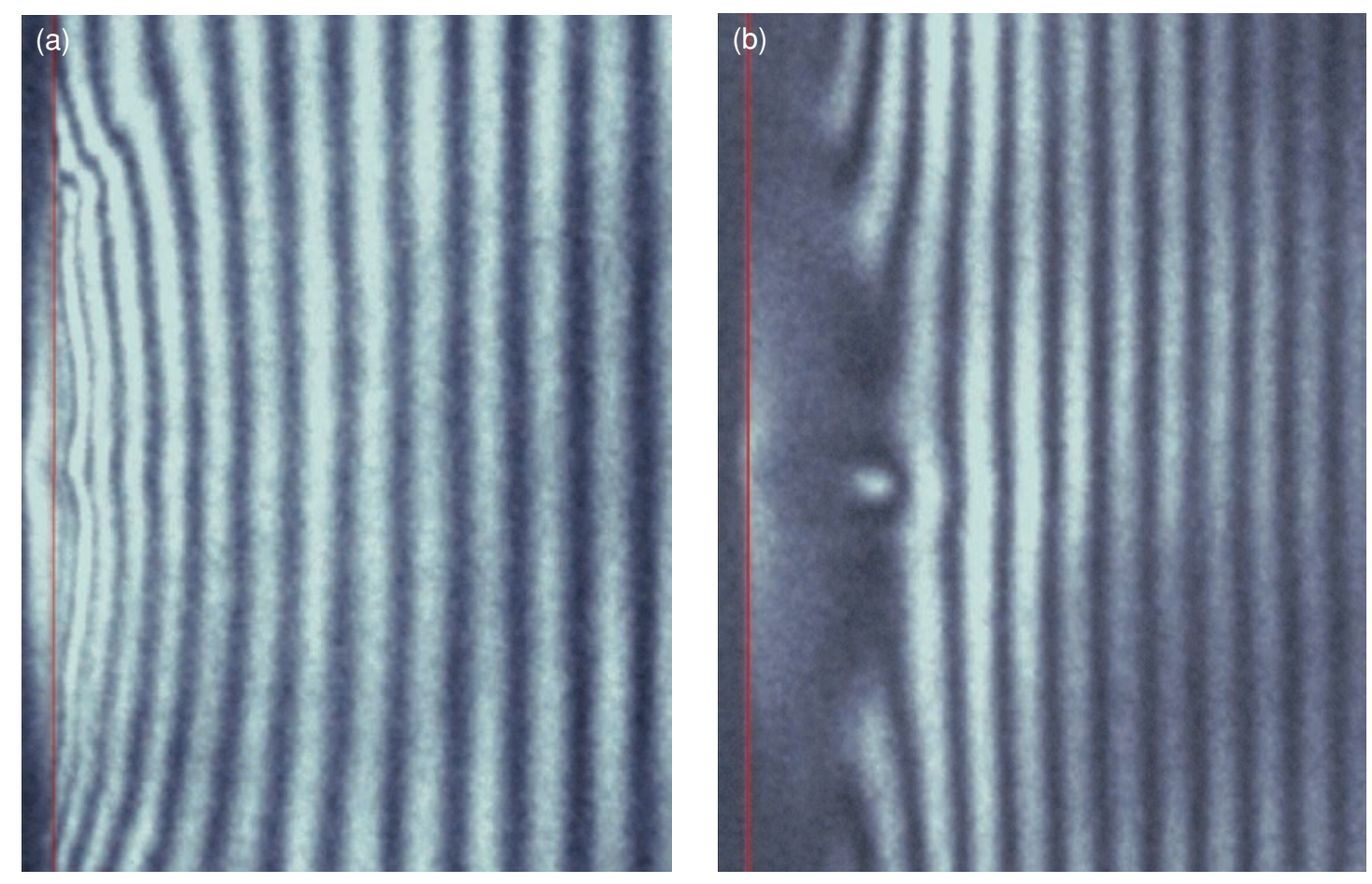

(a) An interferogram of aluminum plasma captured 0.2 nanosecond after the plasma is created shows the expected fringe shifts bending to the right, away from the high-density plasma region. (b) An interferogram captured 3.2 nanoseconds later shows an anomalous negative fringe shift with fringes bending to the left, toward the high-density plasma region.

Reprinted from Science \& Technology Review, November 2005 UCRL-TR-218607 


\section{Electrons: Bound Versus Free}

According to Nilsen, plasma physicists in the past who have used optical lasers to probe plasmas have assumed that the index of refraction, which is used to determine plasma density, can be calculated using only the number of free electrons. Using the significantly shorter wavelength 14.7-nanometer x-ray laser, Nilsen's colleagues probed plasmas many orders of magnitude denser and hotter than those probed using optical lasers. When a plasma's density is measured by x-ray laser interferometry, the interferometer splits the x-ray beam into two paths, with one beam propagating through the plasma and then recombining with the second reference beam. When recombined, the beams display the interference pattern, which is captured by a chargecoupled device camera. The image, or interferogram, appears as a series of alternating light and dark bands, or "fringes," with the fringes bending away from the region of high density.

Fringe shift is calculated as the distance between light bands. For instance, if the bend is equivalent to the distance between two bands, then the number of fringe shifts is 1 . The resulting number of fringe shifts $\left(N_{\text {fringe }}\right)$ is subsequently used in calculations that incorporate other variables, such as wavelength, index of refraction, and number of free electrons, to determine plasma density.

Experimentalists have been using this technique with assumed reliability for many decades. However, occasionally researchers have observed negative fringe shifts - fringes that bend toward the high-density region. "The bands appear," says Nilsen, "as if they are being sucked into a black hole of high-density plasma." Other scientists who have observed this phenomenon thought the anomalies were due to equipment or experimental error. Because they had no explanation for their observations, no findings were published.

That was not the case with Nilsen and his colleagues. "We wanted to figure out why the fringe was bending the wrong way," says Nilsen. "The negative fringe shift implied a negative plasma density, which we knew was not physically possible." The more likely explanation was a greater contribution of bound electrons to the index of refraction. As it turns out, x-ray lasers interact more with bound electrons than do optical lasers. In a small number of experiments using a tabletop x-ray laser on warm, dense plasmas, Nilsen's colleagues found that as the plasma cools, more anomalies appeared in the collected data, confirming the importance of considering bound electrons.

\section{Doing the Numbers}

Nilsen and his colleagues realized that because the contribution of the bound electrons to the index of refraction was so significant, the standard formulas for plasma density needed to be recalculated. So, Nilsen and Livermore physicist Jim Scofield took on the challenge of recalculating the formulas using x-ray interferometry. According to Nilsen, their goal was to calculate the index of refraction for any material at any temperature, density, or photon energy. "We revised the numbers using both theoretical and experimental results."

To date, the new calculations have been incorporated into a number of computer codes, including the Inferno and OPAL codes, which are used to predict such physical characteristics of plasmas as opacity and equations of state. Gaining a better understanding of these characteristics brings scientists closer to myriad insights on subjects from thermonuclear charges to radiation transport to the evolution of stars.

- Maurina S. Sherman

Key Words: bound electrons, free electrons, fringe shifts, index of refraction, plasma density, $\mathrm{x}$-ray interferometry.

For further information contact Joseph Nilsen (925) 422-4766 (nilsen1@IInl.gov) or Jim Dunn (925) 423-1557 (dunn6@IInl.gov).

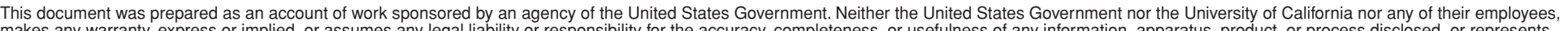

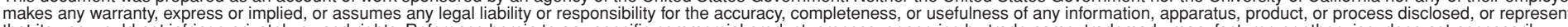

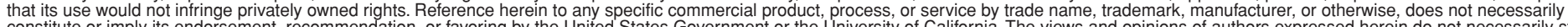

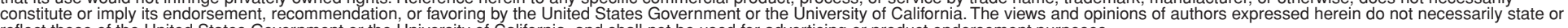
reflect those of the United States Government or the University of California, and shall not be used for advertising or product endorsement purposes.

This work was performed under the auspices of the U.S. Department of Energy by University of California, Lawrence Livermore National Laboratory under Contract W-7405-Eng-48. 